\section{Volumetric SAR near-field upsampling and basebanding}

\section{J. Elgy, D. Andre, and M. Finnis}

Highly sampled imagery offers many benefits to the radar practitioner, ranging from easier image coregistration to simple visual appeal. However, it is often overlooked due to the computational burden forming such an image imposes. Fast image formation typically imposes restrictions on the imaging scenario, for example synthetic aperture radar (SAR) far-field, and exploits parallelism through use of modern multicore architecture. Imposing a SAR near-field requirement on the image formation limits the applicability of several of the faster algorithms, thus there is a need to create a general process to achieve highly sampled imagery, regardless of the imaging regime. In this letter, a method for accurately upsampling near-field (SAR) imagery is presented. This is applicable to both SAR near-field and SAR far-field scenarios. The methodology is discussed, and an example is provided in the form of a SAR near-field volumetric image of a miniature tank. The limitations to the approach are discussed and prospects for future work given.

Introduction: Within the broad field that is synthetic aperture radar (SAR), there is a desire for finely sampled imagery, be it for removing ambiguities, for improved interferometry, or for the simple visual appeal. The limiting factor is the computational time it takes to form such an image.

There are several methods for reducing the computation time, mostly focusing on effective parallelisation by dividing the image into subdomains $[1,2]$, or by utilising different image formation algorithms in each subdomain for example, Doppler Beam Sharpening as opposed to Backprojection [3].

In this letter, a rapid method for reliable SAR resampling, applicable to both SAR near-field and far-field, is introduced. This method is reliant upon a new spatially variant basebanding technique described here, and is demonstrated using a measured volumetric SAR near-field image, formed via standard Backprojection [4].

Spatially Variant Basebanding: The aim of basebanding is to shift the complex spatial frequency support of an image, such that it is centred around the origin, thus reducing overall phase gradient across the image [5]. While conventionally used in interferometry to improve results, it has the additional benefit of reducing the chance of gaps or discontinuities, occurring in the Fourier domain during the upsampling process, and eliminating the need for any ad hoc circular shifting of the data in the frequency domain [6].

It has been shown in $[5,7]$ that conventional basebanding is ineffectual in the SAR near-field regime, herein defined as $d<2 L_{c r}^{2} \lambda_{c}^{-1}$ for an image cross range extent $L_{c r}$ and a centre wavelength $\lambda_{c}$. Here, wavefronts are significantly curved across the scene so that separate areas of the image have significantly different Fourier domain image supports. These sub-supports make the bulk shift used in conventional basebanding sub-optimal. An alternative SAR near-field basebanding approach was proposed in $[5,7]$ which operates on a per pixel basis.

Consider an image geometry defined by a pixel position $\boldsymbol{P}$, the mean synthetic aperture position $\overline{\boldsymbol{T}}$ and the scene centre $\boldsymbol{S}$

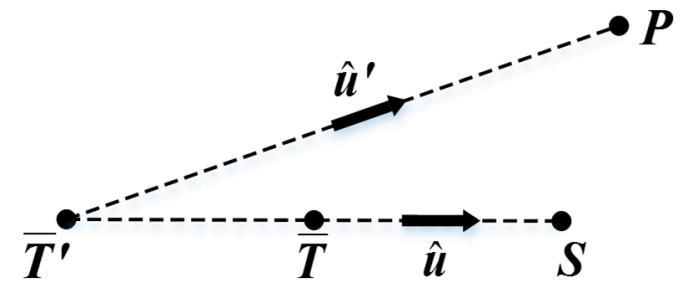

Fig. 1 Simplified image geometry showing the unit vectors necessary for both conventional and spatially variant basebanding.

To centre the spatial frequency image support around the origin, the support is usually shifted, via a phase ramp applied in the image domain, along the unit vector $\widehat{\boldsymbol{u}}$, where $\boldsymbol{u}=\boldsymbol{S}-\overline{\boldsymbol{T}}$, which is not spatially variant over the image. While this works well in the SAR far-field regime, it has been found that in the SAR near-field regime, a spatially variant basebanding implementation produces a substantially reduced phase gradient $[5,7]$. A unit vector $\widehat{\boldsymbol{u}}^{\prime}$ is introduced, which is obtained from $\boldsymbol{u}^{\prime}=\boldsymbol{P}-\overline{\boldsymbol{T}}^{\prime}$, where $\overline{\boldsymbol{T}}^{\prime}=\mathbf{2} \overline{\boldsymbol{T}}-\boldsymbol{S}$. This unit vector $\widehat{\boldsymbol{u}}^{\prime}$ varies with pixel position, and is used in the construction of the basebanding phase screen applied to the image,

$$
\begin{gathered}
R(\boldsymbol{P})=e^{\frac{i 4 \pi f_{c} P_{x} \widehat{u^{\prime} x}}{c}} \times e^{\frac{i 4 \pi f_{c} P_{y} \widehat{u^{\prime}} y}{c}} \times e^{\frac{i 4 \pi f_{C} P_{C} \widehat{u_{z}^{\prime}}}{c}} \\
R(\boldsymbol{P})=e^{\frac{i 4 \pi f_{C}}{c} \cdot \widehat{\boldsymbol{u}^{\prime}}}
\end{gathered}
$$

where $f_{c}$ is the centre frequency for the SAR collection and $c$ is the speed of light. The original complex SAR image is multiplied on a per pixel basis by $R$ to give the new basebanded image. For any given region on the SAR image, this has the effect of shifting the corresponding energy in the Fourier domain along $\widehat{\boldsymbol{u}^{\prime}}$ towards the origin of the spatial frequency domain. Overall, this substantially reduces phase gradients over the SAR image and will compress the overall spatial frequency support so that it has approximately the extent of its far-field equivalent. Equations (1) and (2) are valid for both two and three-dimensional imagery.

To extend the formalism to bistatic geometries, $\widehat{\boldsymbol{u}}^{\prime}$ in (1) and (2) is replaced by the mean of the two $\widehat{\boldsymbol{u}}^{\prime}$ vectors obtained from considering the transmitting and receiving antenna synthetic apertures separately.

\section{Volumetric Upsampling}

Similarly to $[6,8]$, the imagery is upsampled via zero-padding in the spatial frequency domain. First, a coarsely sampled image undergoes basebanding. This image is then Fourier transformed into the spatial frequency domain.

Zero-padding in the frequency domain acts as a sinc interpolation in the image domain, so that by symmetrically zero-padding the dataset to a desired sampling, the image is interpolated. This does not introduce any new information, thus this method cannot be used to improve resolution. Finally, inverting the Fourier transform produces an image that has been upsampled to the desired voxel density.

This process is illustrated in Fig 2.

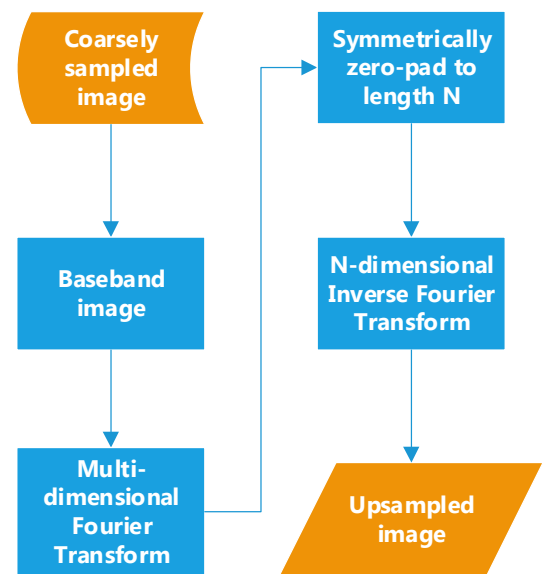

Fig 2 Flowchart illustrating the upsampling process.

For a high quality interpolation result, the initial image should be formed at least in agreement with the Nyquist criterion. This can be achieved by ensuring each voxel, of dimensions $\Delta P_{x}, \Delta P_{y}, \Delta P_{z}$, is the same size or less than a resolution cell. For a broadside collection with horizontal aperture $\phi_{a}$ and vertical aperture $\theta_{a}$, the voxel sizes are as follows,

$$
\begin{gathered}
\Delta P_{y} \leq \frac{c}{2 B_{w}} \\
\Delta P_{x} \leq \frac{c}{4 f_{c} \sin \left(\frac{\phi_{a}}{2}\right)} \\
\Delta P_{z} \leq \frac{c}{4 f_{c} \sin \left(\frac{\theta_{a}}{2}\right)}
\end{gathered}
$$


Where $B_{w}$ is the bandwidth, $\phi_{a}$ and $\theta_{a}$ are the azimuth and polar aperture extent angles.

If the above criterion is met, once appropriately basebanded, the spatial frequency extent of measured data is contained within the unambiguous frequency extent of the image.

Laboratory Data Example: As stated, this approach described above was developed with the aim to improve the computation time for high quality SAR near-field volumetric SAR images.

The approach is demonstrated using a monostatic volumetric SAR image of a $20^{\text {th }}$ scale T72 tank, shown in Fig $4 \mathrm{a}$. Using a $0.5 \mathrm{~m}$ by $0.5 \mathrm{~m}$ synthetic aperture and a $21.5 \mathrm{GHz}$ to $50 \mathrm{GHz}$ frequency range provides resolutions of approximately $5 \mathrm{~mm}$ in the three dimensions. For a full-scale T72 tank, this would be equivalent to $10 \mathrm{~cm}$ resolution.

Converting this image to the Fourier domain reveals that the image support is larger than the unambiguous frequency extent of the image, as seen in figures $3 \mathrm{a}$ and $3 \mathrm{c}$, and therefore wraps around. This is due to the SAR near-field nature of the radar collection, where each patch of the image has its own differently shaped and positioned Fourier domain image sub-support. Applying the spatially variant basebanding, both centres and compresses the overall support, as seen in figures $3 \mathrm{~b}$ and $3 \mathrm{~d}$, leading to the overall support being visibly contained within the unambiguous frequency extent. In effect, the Fourier domain image subsupports have all been centred by this spatially variant basebanding process. a)

c)
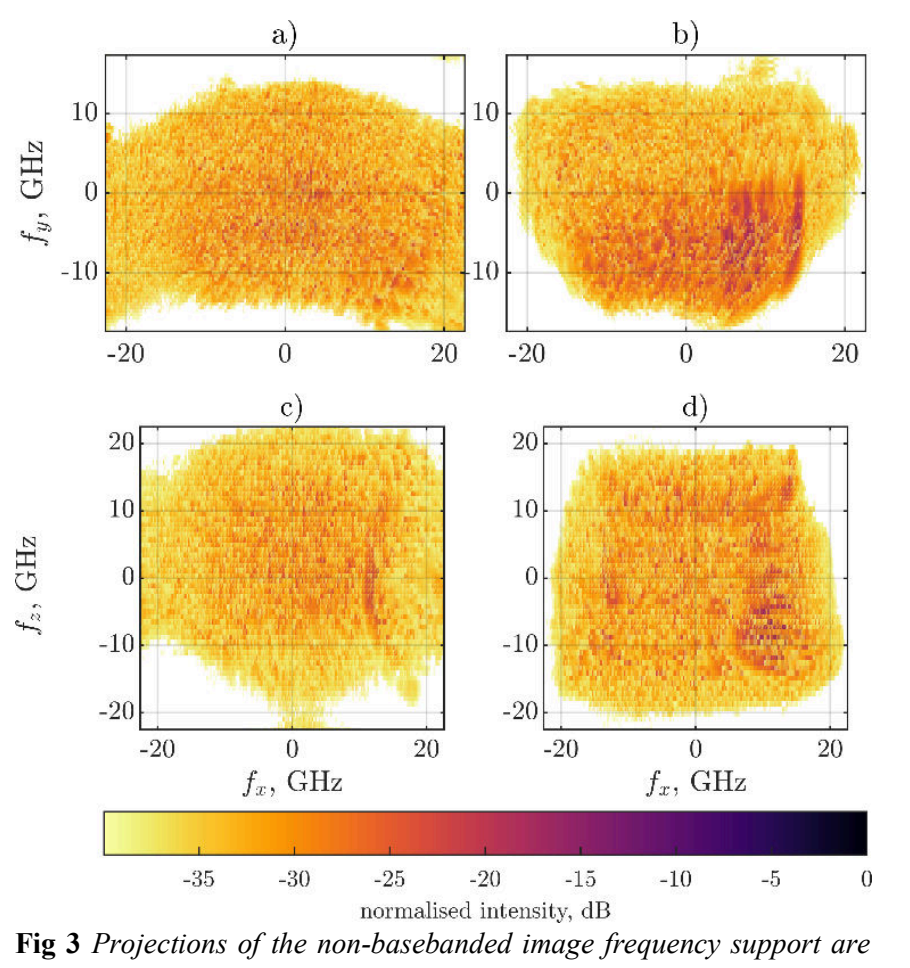

b)

d)
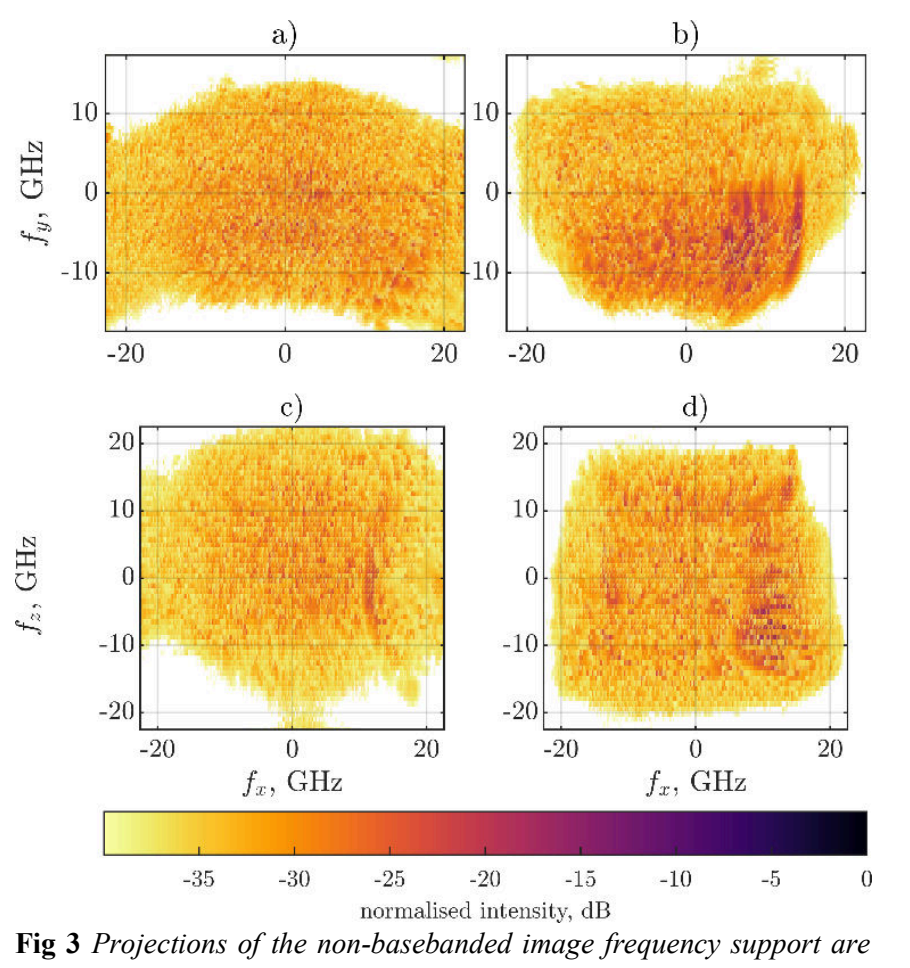

normalised intensity, $\mathrm{dB}$

Fig 3 Projections of the non-basebanded image frequency support are shown on the left in a) and c), whereas those for the basebanded support are shown on the right in b) and d).

Since the basebanded support does not wrap around, it can be padded with zeros without introducing any gaps or sharp discontinuities in the Fourier domain data, avoiding the introduction of image artefacts and maximising the upsampled image quality. Figure 4 shows the result of this upsampling. The spatial frequency has been zero-padded by a factor of 4 in each dimension, resulting in 64 times as many voxels in the time domain image. Figure $4 a$ shows the original coarse image whereas figure $4 \mathrm{~b}$ shows the upsampled version. When zooming in to this high radar resolution image, it can be seen that there is a definite improvement in image quality between Figure $4 \mathrm{a}$ and $4 \mathrm{~b}$, thus validating the approach.
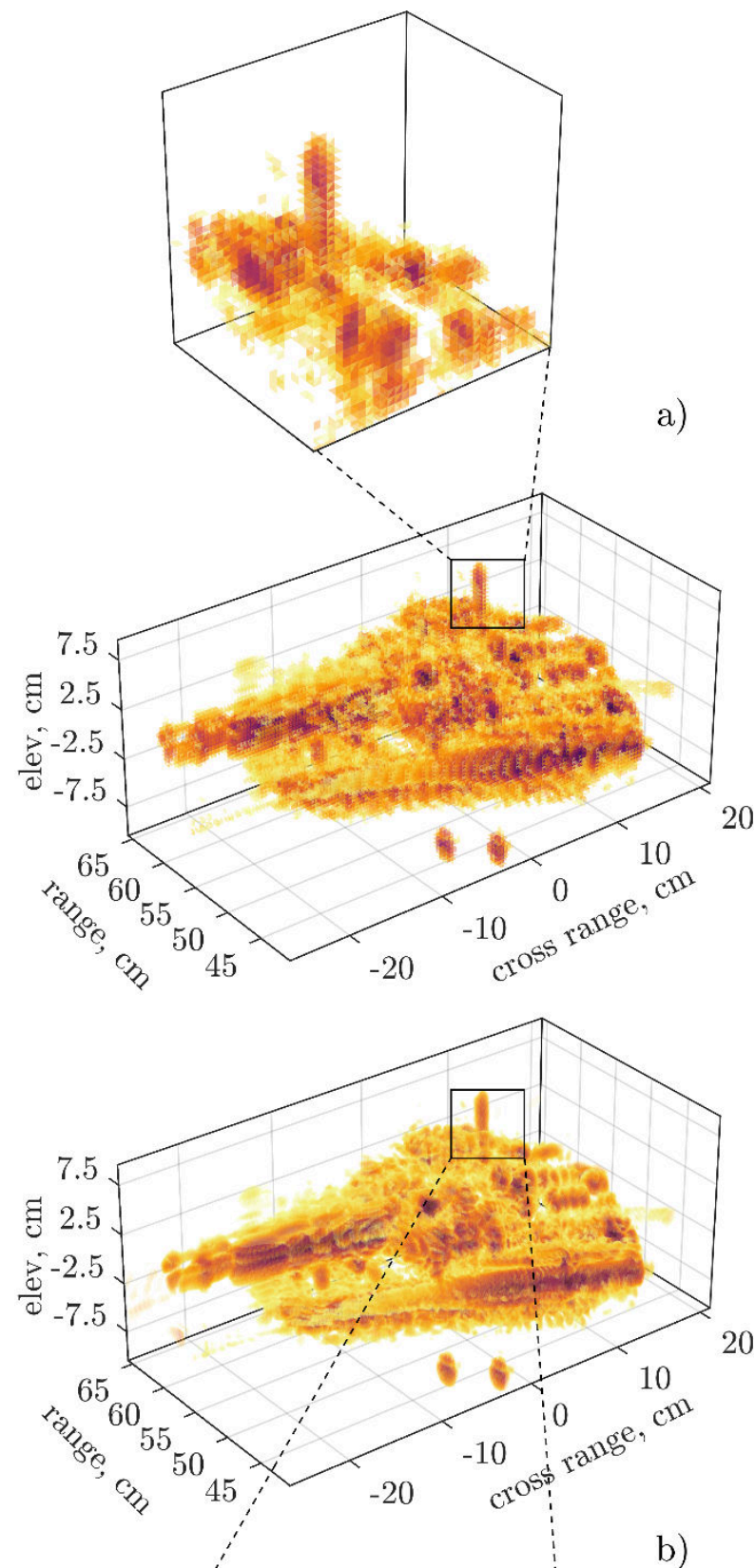

20

b) 
in Fig 5, where the execution time taken to form an $N \times N \times N$ image from 10201 radar measurements, is compared for direct Backprojection and this upsampling method. The computations were carried out using Matlab and two Intel Xeon Gold 12 core processors. The results show that for this example, the upsampling process is significantly faster when $\mathrm{N}$ is sufficiently large: approximately twice as fast when $\mathrm{N}=212$ and ten times as fast when $\mathrm{N}=478$.

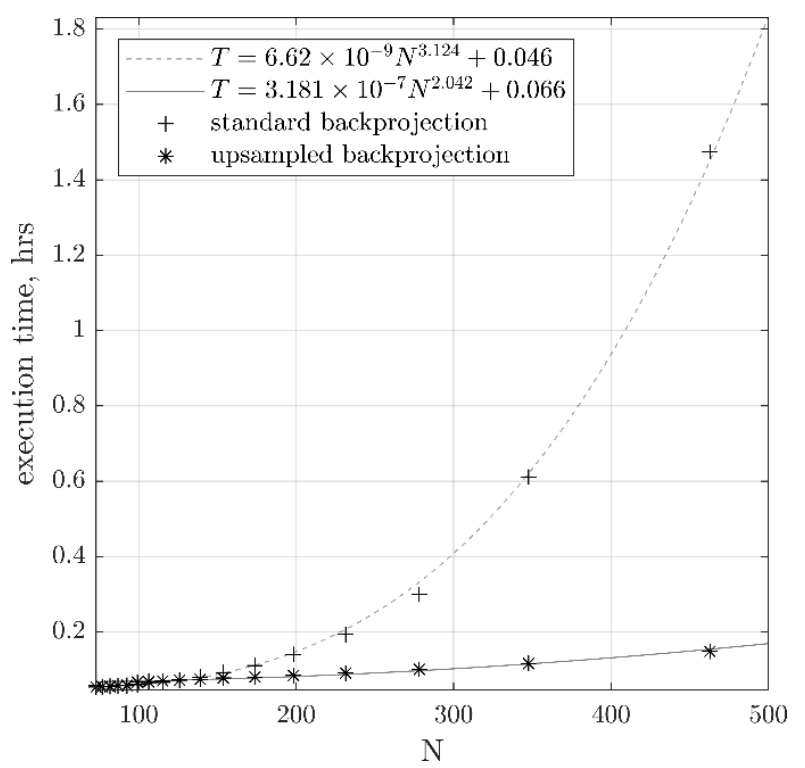

Fig 5 Comparison of the computation times between standard Backprojection and the proposed upsampling process when used to form and $N \times N \times N$ image from 10201 radar pulses.

A best fit curve has been added to both methodologies in the form of $T=$ $A N^{B}+C$. The standard Backprojection algorithm follows a cubic curve ( $\left.A=6.62 \times 10^{-9}, B=3.124, C=0.046\right)$, whereas the upsampling process follows a square curve $\left(A=3.181 \times 10^{-7}, B=2.042, C=\right.$ 0.066 ) and as such, the growth rate of the function is shown empirically to be substantially smaller.

Window Weighting: Due to possible discontinuities between the image spatial frequency support and zero-padded elements of the upsampled image, the upsampling process is susceptible to the Gibb's Phenomenon. A window function is typically applied over the Fourier domain image support to counteract this effect, for example, the power window used in [8]. Such windows, implemented post image formation, are often suboptimal in the SAR near-field due to the large spread of the Fourier domain image support, which can cause it to wrap around the unambiguous frequency extent. Additionally to this however, as noted earlier, in the SAR near-field regime, the overall image support is composed of many sub-supports, each pertaining to a local patch in the image. As these sub-supports are in different locations, without the spatially variant basebanding, the window weighting procedure cannot be effectively implemented post image formation, over all image patches. With spatially variant basebanding however, all the Fourier domain image sub-supports are brought together, so that the window weighting can be effectively implemented, post image formation, over all of them and achieve the desired sidelobe suppression across the whole image.

Conclusions: As a means of achieving highly sampled imagery, the method presented provides an attractive option in terms of achieving faster image formation times. This approach is less restrictive than the prior art in that it operates in both SAR near-field and SAR far-field, although it retains the need to satisfy the Nyquist criterion for the initial image formation.

It is noted that when implementing this approach prior to interferometric processing, one should apply the same phase screen to both SAR images in order to retain the phase relation between them. This phase screen may be calculated from the SAR geometry of one image, and will be then suboptimal for the other image. Alternatively, one can assume a mean SAR geometry for both collections, and calculate the spatially variant phase screen from that.

As well as allowing the zero-pad interpolation process, the spatially variant basebanding described, also allows the application of a window weighting across all the centred Fourier domain image sub-supports, providing sidelobe suppression across the whole image.

In through-wall and ground penetrating radar scenarios, the wavefronts of the electromagnetic pulses are refracted. This refraction can be corrected for by applying the appropriate delay in the image formation [9]; however, this leads to a greater spread in the frequency support of the image. Future work should entail developing the basebanding process, such that it operates optimally through a combination of different media, in order to reduce the sampling rate needed when imaging through inhomogeneous media. E.g. sedimentary layers.

Acknowledgements: The authors would like to thank Keysight for loaning the network analyser used in the data gathering.

J. Elgy and D. Andre (Centre for Electronic Warfare, Information, and Cyber, Cranfield University, Defence Academy of The United Kingdom, Shrivenham, United Kingdom)

Email: James.D.Elgy@Cranfield.ac.uk

M. Finnis (Centre for Defence Engineering, Cranfield University, Defence Academy of The United Kingdom, Shrivenham, United Kingdom)

\section{References}

1. Park, J., Tang, P.T.P., Smelyanskiy, M., Kim, D., Benson, T.: 'Efficient backprojection-based synthetic aperture radar computation with many-core processors', Sci. Program., 2013, 21, (3-4), pp. 165-179

2. Gocho, M., Oishi, N., Ozaki, A.: 'Distributed Parallel Backprojection for Real-Time Stripmap SAR Imaging on GPU Clusters', in 'Proceedings - IEEE International Conference on Cluster Computing, ICCC', Honolulu, HI, USA, September 2017, pp. 619-620

3. Rigling, B., Garber, W., Hawley, R., Gorham, L.: 'Wide-area, persistent SAR imaging: Algorithm tradeoffs' IEEE Aerosp. Electron. Syst. Mag., 2014, 29, (1), pp. 14-20

4. Elgy, J., Andre, D.: 'GBSAR-Proc', https://cord.cranfield.ac.uk/articles/GBSAR-Proc/9693581, accessed November 2019

5. Andre, D., Blacknell, D., Finnis, M.: 'An Improved SAR-Near-Field Spatially Variant Basebanding Technique', 'Proceedings of the Institute of Acoustics' Lerici, Italy, September 2018, pp. 173-180

6. El-Darymli, K., McGuire, P., Power, D., Moloney, C.: 'An algorithm for upsampling spotlight SAR imagery: a Radarsat-2 SLC perspective', in 'Algorithms for Synthetic Aperture Radar Imagery XX' Baltimore, MD, USA, May 2013, p. 874607

7. Andre, D., Morrow, I.L.: 'A new spatially variant SAR basebanding technique', in 'EUSAR 2018; 12th European Conference on Synthetic Aperture Radar' Aachen, Germany, June, 2018, pp. 1066-1069

8. El-Darymli, K., McGuire, P., Gill, E., Power, D., Moloney, C.: 'Errata: Effect of detection on spatial resolution in synthetic aperture radar imagery and mitigation through upsampling' J. Appl. Remote Sens., 2014, 8, (1), p. 089997

9. Elgy, J., Andre, D., Finnis, M., Blacknell, D.: 'Data Driven Corrections to Multistatic 3D Through-Wall Radar Imagery', in 'Proceedings of the Institute of Acoustics' Lerici, Italy, September 2018, pp. 199-208 